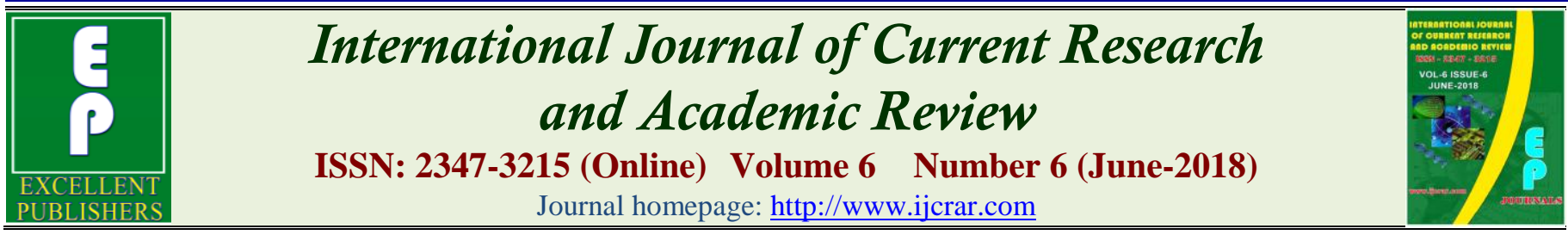

doi: https://doi.org/10.20546/ijcrar.2018.606.012

\title{
Pavlik Harness in Treatment of Developmental Dysplasia of the Hip Joint a Prospective Short Term Follow up Clinical Study
}

\author{
Hadi Kareem Hadi ${ }^{*}$, Dhafer Fouad and Zaid Ali Jasim Shaban
}

AL-Wasity Teaching Hospital, Baghdad-Iraq

*Corresponding author

\section{Abstract}

The Pavlik harness is the first non-invasive line of treatment of newborn with developmental dysplasia of the hip that keeping the epiphysis within the acetabulum, allowing some movement and a good perfusion, to avoid the avascular necrosis of head of femur. The aim of this study is to assess the success of treatment with Pavlik harness in infants with developmental dysplasia of the hip. Therefore, 50 patients younger than six months of age with bilateral or unilateral developmental dysplasia of the hip (DDH) that treated by Pavlikharness at Al-Wasty Teaching Hospital and followed up for 3 weeks were enrolled. Theresponse to treatment was monitored weekly by ultrasound examination. Among the studied group, 42(86\%), showed good response to treatment during the period of follow up. High rate of success reported with unilateral developmental dysplasia of the hip and in patient who started treatment within first week of life. Side of pathology, gender had no effect on successfulness of treatment. Avascular necrosis not reported with our cases. Hence, in conclusion Pavlik harness appeared to be a safe and successful method for treatment of developmental dysplasia of the hip
\end{abstract}

\section{Introduction}

The term developmental dysplasia of the hip (DDH) referred to the all deformities associated with growing of the hip joint that includes the subluxation, frank dislocation, instability and dysplasia of femoral head and acetabulum (Guille et al., 2000). The DDH is a disorder of multiple causative factors such as familial tendency, racial predilection, gender and factors of mechanical origin that related to prenatal and postnatal periods (Herring, 2008).

As long as the proper development of the hip joint in childhood depend on the femoral head stability within the acetabulum so the accurate management of unstable joint play an important in the determination of the prognosis of $\mathrm{DDH}$, as the best possible functional outcome was obtained with earlier diagnosis and management (Atalar et al., 2007).

Sonography considered suitable method for diagnosis of dysplasia of hip joint within the earlier months or days of Childs life where the decision of treatment if it is necessary or not will rely on Graf's classification (Paton et al., 2004).

The overwhelming complication of iatrogenic origin with developmental dysplasia of the hipis avascular necrosis of femoral head, which is potentially devastating and often-untreatable complication (Bialik, 
2007). The objectives of treatment the DDH is to get concentrically reduced stable hip joint at an earliest possible age with minimal incidence of complications (Lipton et al., 2007). The available braces for stabilizing the hip joint with developmental dysplasia of the hipare the Craig splint, Von Rosen splint and Pavlik harness but the last device which is first used by the Pavlik harness in 1944, is the most commonly used today to treat this condition (Choudry et al., 2013). The Pavlik harness allows for reduction spontaneously without rigid fixation, allowed ultrasound observation of the reduction, allows diapers to change without removal of brace, inexpensive and easy inusing (Vitale and Skaggs, 2001).

The using of Pavlik harness after the of six months of age was not recommended for management of developmental dysplasia of the hip by most authors (Ömeroğlu et al., 2015).

A retrospective study was conducted by (Pollet et al., 2010) showed encouraging results with patients starting treatment at the age of 6-24 months of age.

\section{Patients and Methods}

From first.Jan.-31 Dec. 2017, 50infant (28 female, 22 male), 8 with bilateral and 42 withunilateral DDH who were referred to us from different pediatric Hospitals and treated by using the Pavlik harness at Al-Wasity Teaching Hospital was included in this study.Patients with; teratological dislocation,six months of age or more and whom had multiple congenital deformitieswas ruled out of the study. After the using of Pavlik, harness the ultrasound used weekly by same specialist for assessment of position of hip joint to get more results that are reliablebut no dynamic assessment performed after starting of treatment. When the hip starting reduction concentrically; we starting in removal of harness (firstly for one hour per day and period of removal increased gradually). The child reviewed at six and twelve weeks of age, then at 6 months and 12 months of age. The hip development was assed radiographically when the ossification nuclei established.

The harness treatment discontinued if there was no improvement by the third week of follow-up. Statistical analysis; frequency and percentage used to represent the categorical data, chi-square test used to assess the significance of comparison in categorical variables, Fisher's exact test used as an alternative when chi square test was inapplicable, level of significance (P. value) was set at $\leq 0.05$ as cutoff to be considered as significant.

\section{Results and Discussion}

The results showed that $60 \%$ of patients belonged to age group of less than seven days, 54\% with right side involvement, $90 \%$ with unilateral involvement and $84 \%$ responded to treatment successfully as seen in (Table $1 \&$ Fig.1).

The successful treatment was affected by the age at treatment was started where the patients who started treatment within first week of their age showed better response rate. This association was statistically significant $(\mathrm{p}=0.04)$ as displaced in (Table 2$)$.

The results revealed that the patients with bilateral DDH showed a lower rate of successin comparison to those with unilateral $\mathrm{DDH}$. The association was statistically significant $(\mathrm{P}=0.020)$, (Table 3$)$.

Furthermore, our data indicated that the gender and side of pathology has no effect on success of treatment. Avascular necrosis not reported with our cases.

Follow-up at weekly intervals with Pavlik harness is necessary. Other treatment modalities should considerif the reduction of the hip joint is failed after three week of treatment (Atalar et al., 2007). According to (Kitoh et al., 2009) and (Tiruveedhula et al., 2015) the acetabular dysplasia will aggravated by continuation of treatment with harness in dislocated joint ("Pavlik harness disease") and that will increase the difficulty of subsequent closed reduction, in addition the probability of avascular necrosis will be increased.

Author (Cashman et al., 2002) concluded that the incidence rate of residual dysplasia of acetabulum after right treatment with harness of Pavlik could not disregarded. The author (David et al., 2015) in their study found a $4 \%$ recurrent dysplasia after successful treatment of DDH so they recommended follow-up to 6 month of infant age. (Song et al., 2008) reported that the hipdysplasia of mild severity could develop as the child grows older even with successful treatment by Pavlik harness.

Our data indicated that the success rate was $84 \%$ and this rate was lower than that reported by (Taylor et al., 1997) as they got 95.7\% successful reduction with Pavlik harness in their cases. Similar results was reached by (Grill et al., 1988) as they got $95.35 \%$ healing rate in infant with dysplastic hips but they observed Avascular necrosis of the femoral head in $2.38 \%$ of their cases. 
Table.1 Baseline characteristics and status of outcome of studied group

\begin{tabular}{llll}
\hline Variable & & No. & \% \\
\hline Age & $<7$ days & 30 & $60.0 \%$ \\
\cline { 2 - 4 } & $\geq 7$ days & 20 & $40.0 \%$ \\
\hline Side of involvement & Left & 23 & $46.0 \%$ \\
\cline { 2 - 4 } & Right & 27 & $54.0 \%$ \\
\hline Status of involvement & Bilateral & 5 & $10.0 \%$ \\
\cline { 2 - 4 } & Unilateral & 45 & $90.0 \%$ \\
\hline
\end{tabular}

Table.2 Response status according to age of starting the treatment

\begin{tabular}{|c|c|c|c|}
\hline \multirow[b]{2}{*}{ Age } & \multicolumn{2}{|c|}{ Response status } & \multirow{2}{*}{ Total } \\
\hline & Respondn (\%) & Not respondn (\%) & \\
\hline$<7$ days & $28(93.3)$ & $2(6.7)$ & $30(100.0)$ \\
\hline$\geq 7$ days & $14(70.0)$ & $6(30.0)$ & $20(100.0)$ \\
\hline Total & $42(84.0)$ & $8(16.0)$ & $50(100.0)$ \\
\hline
\end{tabular}

$P$. value $=0.040$

Table.3 Response status according to age of starting the treatment

\begin{tabular}{lccc}
\hline & \multicolumn{2}{c}{ Response status } & Total \\
\cline { 2 - 3 } Status of involvement & Respondn $(\%)$ & Not respondn (\%) & \\
\hline Bilateral & $2(40.0)$ & $3(60.0)$ & $5(100.0)$ \\
\hline Unilateral & $40(88.9)$ & $5(11.1)$ & $45(100.0)$ \\
\hline Total & $42(84.0)$ & $8(16.0)$ & $50(100.0)$ \\
\hline$P$. value $=\mathbf{0 . 0 2 0}$ & & &
\end{tabular}


Fig.1 Distribution of response to treatment among the studied group

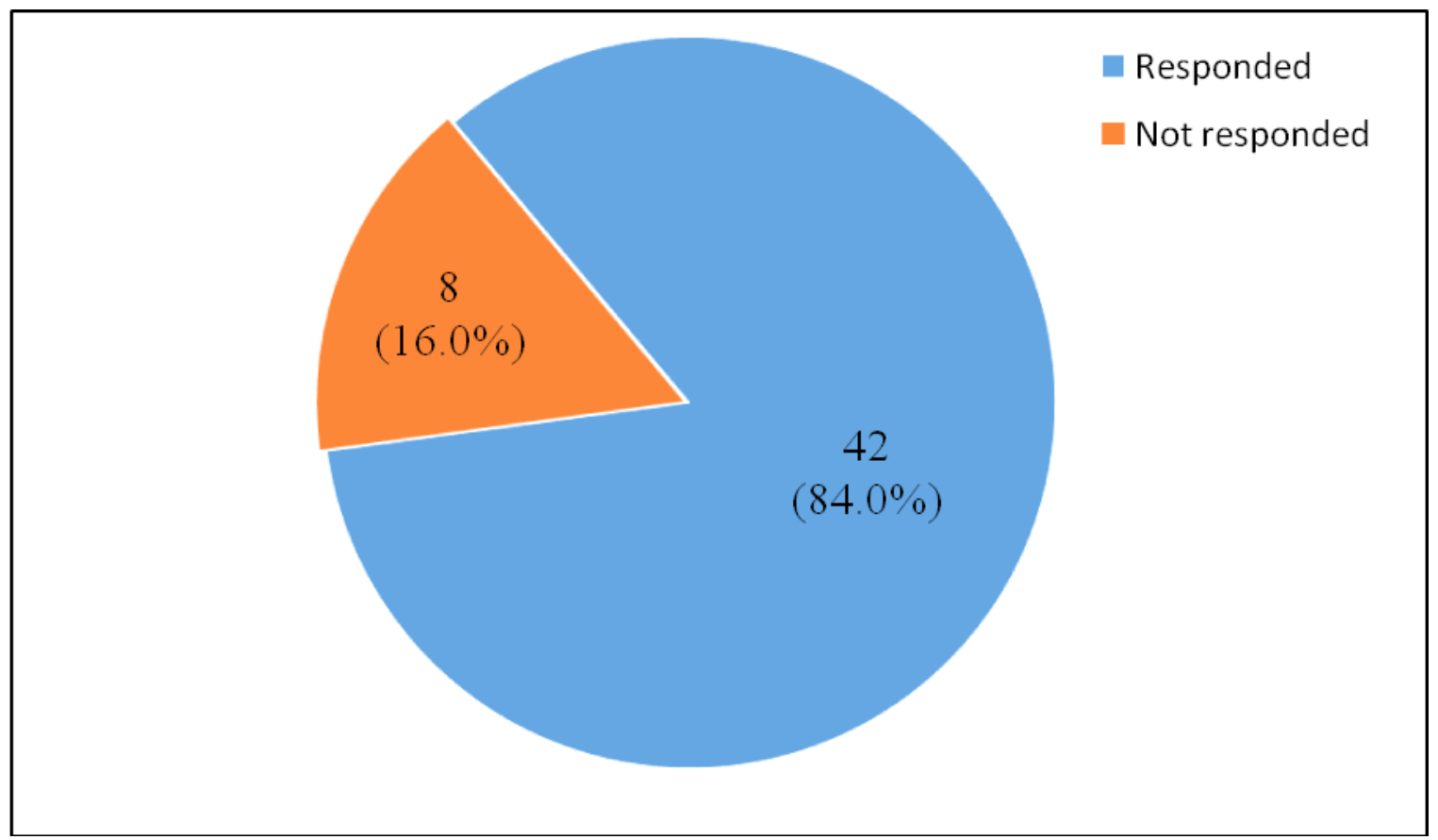

The difference in success rate with other studies might explained partly on the bases of difference in culture and education of families and to what extent they follow the instructions of the treating physician regarding the suitable period of daily removal of harness

We found there was significant association between the statues of Outcome of treatment and the age of starting the treatment as well as with status of DDH wheather bilateral or unilateral where the high rate of failure was reported with the patients that starting treatment at second week of their age and with bilateral DDH.

Same significant association with these variables also found by (Atalar et al., 2007) in their study as they also reported high rate of failure in patients with bilateral DDH and in the patientsaged 8 weeks and over. In conclusion, Pavlik harness is safe and effective method for treatment of developmental dysplasia of the hip joint.

\section{Ethical issues}

The study protocol was approved by the local committee of ethical issues of the Hospital. All patients' parents/caregiver were informed about the nature and aims of the study and written signed consent were obtained from all patients' parents/caregiver. Data of patients were collected according to the declaration of Helsinki 2000.
Conflict of Interest: None

Funding: Self-funded by the authors

\section{References}

Atalar H Sayli U, Yavuz O Y et al., Indicators of successful use of the Pavlik harness in infants with developmental dysplasia of the hip. Int Orthop. 2007; 31(2): 145-150

Bialik V. Pavlik's method in developmental dysplasia of the hip. Acta Orthop Traumatol Turc. 2007; 41 Suppl 1: 19-24

Cashman JP, Round J, Taylor G et al., The natural history of developmental dysplasia of the hip after early-supervised treatment in the Pavlik harness. A prospective, longitudinal follow-up. J Bone Joint Surg Br. 2002; 84: 418-425

Choudry Q, Goyal R, Paton RW. Is limitation of hip abduction a useful clinical sign in the diagnosis of developmental dysplasia of the hip? Arch Dis Child. 2013; 98: 862-866

David M, Robb C, Jawanda S et al., Late recurrence of developmental dysplasia of the hip following Pavlik harness treatment until normal ultrasound appearance. J Orthop. 2015; 12: 81-85

Grill $\mathrm{F}$ et al., The Pavlik harness in the treatment of congenital dislocating hip: report on a multicenter 
study of the European Paediatric Orthopaedic Society J Pediatr Orthop. 1988; 8(1): 1-8.

Guille JT, Pizzutillo PD and MacEwen GD. Development dysplasia of the hip from birth to six months. J Am AcadOrthop Surg. 2000; 8: 232-242

Herring JA. Tacdjian's pediatric orthopedics. Philadelphia: Saunders Elsevier; 2008.

Kitoh H, Kawasumi M, Ishiguro N. Predictive factors for unsuccessful treatment of developmental dysplasia of the hip by the Pavlik harness. J Pediatr Orthop. 2009; 29: 552-557.

Lipton GE, Guille JT, Altiok H et al., A reappraisal of the Ortolani examination in children with developmental dysplasia of the hip. J Pediatr Orthop. 2007; 27: 27-31

Ömeroğlu H, Köse N, Akceylan A. Success of Pavlik Harness Treatment Decreases in Patients $\geq 4$ Months and in Ultrasonographically Dislocated Hips in Developmental Dysplasia of the Hip. Clin Orthop Relat Res. 2015: Epub ahead of print
Paton RW, Hopgood PJ, Eccles K. Instability of the neonatal hip: the role of early or late splint age. Int Orthop. 2004; 28: 270-273

Pollet V, Pruijs H, Sakkers R et al., Results of Pavlik harness treatment in children with dislocated hips between the age of six and twenty-four months. J Pediatr Orthop. 2010; 30: 437-442

Song FS, McCarthy JJ, MacEwen GD et al., The incidence of occult dysplasia of the contralateral hip in children with unilateral hip dysplasia. J Pediatr Orthop. 2008; 28: 173-176.

Taylor G R, Clarke N M P. Monitoring the treatment of developmental dysplasia of the hip with the pavlik harness the role of ultrasound $\mathrm{j}$ bone joint surg $[\mathrm{Br}]$ 1997; 79-B: 719-23.

Tiruveedhula M, Reading IC, Clarke NM. Failed Pavlik harness treatment for DDH as a risk factor for avascular necrosis. J Pediatr Orthop. 2015; 35: 140143

Vitale MG, and Skaggs DL. Developmental dysplasia of the hip from six months to four years of age. J Am AcadOrthop Surg. 2001; 9: 401-411

\section{How to cite this article:}

Hadi Kareem Hadi, Dhafer Fouad and Zaid Ali Jasim Shaban. 2018. Pavlik Harness in Treatment of Developmental Dysplasia of the Hip Joint a Prospective Short Term Follow up Clinical Study. Int.J.Curr.Res.Aca.Rev. 6(6), 88-92. doi: https://doi.org/10.20546/ijcrar.2018.606.012 